\title{
Latency after Preterm Prelabor Rupture of the Membranes: Increased Risk for Periventricular Leukomalacia
}

\author{
Annick Denzler, ${ }^{1}$ Tilo Burkhardt, ${ }^{1}$ Giancarlo Natalucci, ${ }^{2}$ and Roland Zimmermann ${ }^{1}$ \\ ${ }^{1}$ Department of Obstetrics, Zurich University Hospital, Frauenklinikstraße 10, 8091 Zurich, Switzerland \\ ${ }^{2}$ Department of Neonatology, Zurich University Hospital, Frauenklinikstraße 10, 8091 Zurich, Switzerland
}

Correspondence should be addressed to Tilo Burkhardt; tilo.burkhardt@usz.ch

Received 5 March 2014; Revised 3 June 2014; Accepted 21 June 2014; Published 17 July 2014

Academic Editor: Rosa Corcoy

Copyright (C) 2014 Annick Denzler et al. This is an open access article distributed under the Creative Commons Attribution License, which permits unrestricted use, distribution, and reproduction in any medium, provided the original work is properly cited.

Objective. To identify the risk factors for cystic periventricular leukomalacia (cPVL) and their implications for deciding between immediate delivery and conservative management of preterm prelabor rupture of the membranes (pPROM). Methods. The following risk factors were compared between cPVL infants and 6440 controls: chorioamnionitis, sex, gestational age (GA), birth weight, pPROM, and pPROM-delivery interval. Factor impact on cPVL risk and clinical decision-making was determined by multivariate logistic regression. Results. Overall cPVL prevalence $(n=32)$ was $0.99 / 1000$ births. All cPVL infants but one were born $<34$ weeks of gestation and were $<2500 \mathrm{~g} ; 56 \%$ had histological chorioamnionitis versus $1.1 \%$ of controls (OR 35.9; $95 \%$ CI 12.6-102.7). Because chorioamnionitis is a postnatal diagnosis, logistic regression was performed with prenatally available factors: pPROM-delivery interval $>48$ hours (OR 9.0; 95\%-CI 4.1-20.0), male gender (OR 3.2; 95\%-CI 1.4-7.3). GA was not a risk factor if birth weight was included. Risk decreased with increasing fetal weight despite a prolonged pPROM-delivery interval. Conclusion. pPROM-delivery interval is the single most important prenatally available risk factor for the development of cPVL. Immediate delivery favors babies with chorioamnionitis but disfavors those with non infectious pPROM. In the absence of clinical chorioamnionitis fetal weight gain may offset the inflammatory risk of cPVL caused by a prolonged pPROM-delivery interval.

\section{Introduction}

Cerebral palsy includes a group of nonprogressive movement disorders due to brain lesions or abnormalities in early development [1]. Its prevalence of 2 per 1000 newborns overall rises to 77 per 1000 preterms born at below 28 0/7 weeks of gestation [2,3]. A major cause is cystic periventricular leukomalacia (cPVL) comprising necrosis and subsequent cyst formation of the periventricular white matter: $60-100 \%$ of children with cPVL develop cerebral palsy [4-6]. Although the etiology and pathogenesis of cPVL remain unelucidated, several perinatal risk factors appear involved [7]. Birth asphyxia is no longer assumed the principal culprit [8].

Chorioamnionitis is thought to provoke a fetal inflammatory response syndrome associated with increased fetal cytokines that may lead to neonatal brain injury. Several studies indicate that the cytokines can themselves damage white matter without bacteremia being required [8-15]. An important predictor of chorioamnionitis is preterm prelabor rupture of membranes (pPROM) [16]. One-third of women with pPROM have positive amniotic fluid cultures [17]. Chorioamnionitis is quite common and often subclinical: fever and inflammatory marker elevation are rare in the early stages, making diagnosis difficult. Against this background the optimal management of pPROM remains unknown. The risks of prematurity from immediate delivery have to be balanced against those of ascending intrauterine infection and its probable consequences. Moreover subclinical chorioamnionitis is believed to cause PPROM. At a gestational age below 34 0/7 weeks, half the gynecologists in Australia and New Zealand preferred to induce labor, while the other half chose conservative management [18]. Several studies recommend an active management after 30 weeks $[19,20]$. A Cochrane review from 2010 found no evidence about which strategy is favorable [21]. Despite a lack of randomized studies [22] new British and German guidelines advise active management before 34 weeks and active management between 34 and 36 weeks. Zurich University Hospital has 
hitherto favored conservative management, delaying delivery until clinically mandatory, on the grounds that the higher mortality and morbidity of newborns at lower gestational age are proven whereas the effect of increasing cPVL risk by prolonging pregnancy remains unknown. Only a prospective randomized trial can provide a definite answer. The more limited objectives of the present study were to identify the risk factors for PVL in the conservative pPROM management setting and determine whether prolonging gestation outweighs the risk of $\mathrm{CPVL}$ due to chorioamnionitis.

\section{Materials and Methods}

The study population comprised all babies with cPVL born in Zurich University Hospital's obstetric department between 1993 and 2008. Cranial ultrasound was obtained in infants with gestational age below 32 0/7 weeks or birth weight below $1500 \mathrm{~g}$ at days 1,3 , and 7 of life and repeated weekly until hospital discharge. cPVL was defined according to de Vries et al. [23]. All 6440 infants born between 2005 and 2007 and not affected by PVL served as controls.

During the study period women with premature contractions received tocolytic drugs (hexoprenaline only until 2001, nifedipine or hexoprenaline from 2002 to 2008) for 48 hours to allow lung maturation with $24 \mathrm{mg}$ of betamethasone. Urinary tract infection or bacterial vaginosis was treated with antibiotics (co-amoxiclav or clindamycin). Steroids were repeated every 10 days until 2002. Since then, all women with threatened preterm delivery have received a single course of steroids. Tocolysis was maintained thereafter if contractions recurred after stopping tocolysis. Management of pPROM pregnancy was largely consistent with British Greentop Guideline no. 44 [17]. After pPROM co-amoxiclav was used until 2001 when it was changed to erythromycin [24], chorioamnionitis was monitored using blood tests (including leukocytes and C-reactive protein (CRP) 12 hourly for the first 48 hours ), maternal temperature, and fetal heart rate. Clinical chorioamnionitis $(\geq 3$ of following markers: leukocytes $>20,000 / \mu \mathrm{L}, \mathrm{CRP}>40 \mathrm{mg} / \mathrm{dL}$, maternal temperature $>38^{\circ} \mathrm{C}$, maternal tachycardia $>100 \mathrm{bpm}$, and fetal tachycardia $>160 \mathrm{bpm}$ ) was treated with antibiotics (coamoxiclav) and prompt delivery. If the diagnosis was uncertain, delivery was deferred until chorioamnionitis became clinically obvious or delivery could be delayed no longer for other reasons. Diagnosis was based on placental histology, positive amniotic fluid cultures sampled at cesarean section, or clinical parameters.

Babies born below 25 0/7 weeks were excluded in both groups because in most instances neonatal care was restricted to comfort care. Infants with CPVL were monitored for longterm follow-up. Neurodevelopmental disability was classified according to Palisano et al. [25]. The risk factors recorded in both groups were chorioamnionitis, pPROM-delivery interval, gestational age at delivery, birth weight, gender, race, and parity.

All statistical analyses were performed with STATA 10 Statistics/Data Analysis Software (Stata Corporation, College Station, TX) using Pearson's $\chi^{2}$ test for comparisons of

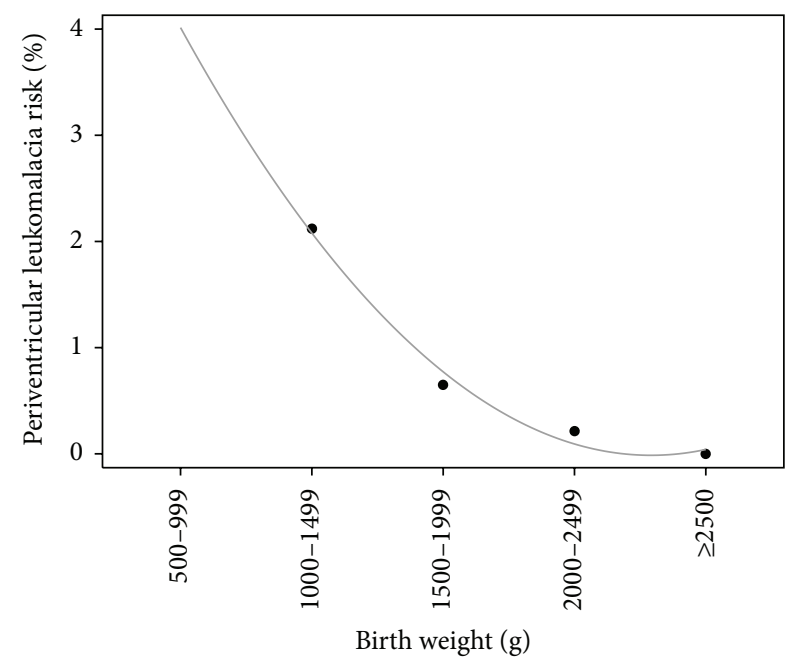

FIGURE 1: Exponential decrease in periventricular leukomalacia risk with increasing birth weight.

frequencies and Wilcoxon's rank-sum test for group comparisons. Odds ratios (OR) with $95 \%$ confidence intervals (CI) were calculated. Subsequent to univariate analysis, multivariate logistic regression was performed to test the impact of factors such as chorioamnionitis, gestational age, birth weight, gender, and pPROM-delivery interval on the incidence of $\mathrm{CPVL}$. The results were used to calculate the risks of developing cPVL at different fetal weights and pPROMdelivery intervals.

Given that the analysis was of anonymized data, the study was exempt from local institutional review board approval. Follow-up data of study preterms below 32 0/7 weeks were extracted from the prospective national database of the Swiss Neonatal Network \& Follow-up Group. According to the recommendations of our Research Ethics Committee the investigators were obliged to inform parents about the scientific use of anonymized data. Parents had the right to refuse participation of their child.

\section{Results}

Between 1993 and 2008, 32,276 infants were born at Zurich University Hospital, including 6027 (18.7\%) preterms (below 37 0/7 weeks); over the same period 32 cases of cPVL were recorded, representing an overall prevalence of $0.99 \%$ (Tables 1 and 2).

cPVL prevalence among preterm infants was $5.3 \%$. All 32 infants with PVL were delivered preterm and all but one before 34 0/7 weeks. cPVL risk decreased exponentially with increasing birth weight (Figure 1) and increasing gestational age. Males were 3 times more affected than females (male : female ratio $24: 8$ ). All birth weights in newborns with cPVL were less than $2500 \mathrm{~g}$ (Table 1). The individual pPROMdelivery intervals of all PVL cases are shown in Figure 2.

Median infant age at cPVL diagnosis was 19 days (3-40 days). Of the 32 infants, five (16\%) died within the first 6 weeks after birth. Of the 27 surviving infants, three (11\%) 
TABLE 1: Baseline cystic periventricular leukomalacia (cPVL) and control group characteristics.

\begin{tabular}{|c|c|c|c|}
\hline Characteristic & $\operatorname{cPVL}(n=32)$ & Controls $(n=6,440)$ & $P$ \\
\hline Gestational age $(\mathrm{d})$ & $207.5(184-255)$ & $273(168-297)$ & $<0.001$ \\
\hline Below 34 gestational weeks (\%) & 97 & 9 & $<0.001$ \\
\hline Multiparous $(n[\%])$ & $12(38)$ & $3145(49)$ & 0.198 \\
\hline Birth weight (g, median [range]) & $1197.5(740-2250)$ & $3270(300-5700)$ & $<0.001$ \\
\hline $\operatorname{PROM}(n[\%])$ & $18(56)$ & $714(11)$ & $<0.001$ \\
\hline pPROM-delivery interval ( $h$, median [range]) & $38(0-960)$ & $1.3(0-2170)$ & 0.004 \\
\hline Chorioamnionitis $(n[\%])$ & $18(56)$ & $73(1)$ & $<0.001$ \\
\hline Lung maturation administration $(n[\%])$ & $27(84)$ & $281(5)$ & $<0.001$ \\
\hline \multicolumn{4}{|l|}{ Infant sex $(n[\%])$} \\
\hline Female & $8(25)$ & 3049 (47) & 0.012 \\
\hline Male & $24(75)$ & $3390(53)$ & \\
\hline
\end{tabular}

PROM: premature rupture of the membranes; pPROM: preterm premature rupture of the membranes.

TABLE 2: Characteristics and management of the cystic periventricular leukomalacia (cPVL) group $(n=32)$ by study period.

\begin{tabular}{|c|c|c|c|}
\hline Characteristic & $\begin{array}{c}\text { Time period } \\
1993-2001\end{array}$ & $\begin{array}{l}\text { Time period } \\
2002-2008\end{array}$ & $\begin{array}{c}\text { Overall } \\
1993-2008\end{array}$ \\
\hline \multicolumn{4}{|c|}{ Chorioamnionitis } \\
\hline Yes & $12(60)$ & $6(50)$ & $18(56)$ \\
\hline No & $6(30)$ & $6(50)$ & $12(38)$ \\
\hline Unknown & $2(10)$ & $0(0)$ & $2(6)$ \\
\hline \multicolumn{4}{|c|}{ Premature rupture of membranes } \\
\hline Yes & $11(55)$ & $7(58)$ & $18(56)$ \\
\hline No & $9(45)$ & $5(42)$ & $14(44)$ \\
\hline \multicolumn{4}{|c|}{ Antenatal betamethasone } \\
\hline Yes & $15(75)$ & $12(100)$ & $27(84)$ \\
\hline No & $5(25)$ & $0(0)$ & $5(16)$ \\
\hline \multicolumn{4}{|l|}{ Infant sex } \\
\hline Male & $15(75)$ & $9(75)$ & $24(75)$ \\
\hline Female & $5(25)$ & $3(25)$ & $8(25)$ \\
\hline Total & $20(1.3 \%$ o $)$ & $12(0.7 \%$ o $)$ & $32(0.95 \%$ o $)$ \\
\hline
\end{tabular}

Data are $n(\%)$.

were lost to follow-up (parental refusal), while $24(89 \%)$ were neurodevelopmentally assessed at a median (range) age of 3.8 years (2.0-10.2 years): one (4\%) was normal, nine $(38 \%)$ were moderately disabled, defined as cerebral palsy grade $<3$ according to the Gross Motor Function Classification System (GMFCS), or cognitive impairment with developmental quotient $55-69$, or moderate visual or hearing impairment, and 14/24 (54\%) were severely disabled, defined as disabling cerebral palsy (grade 3-5 GMFCS), or severe cognitive disability with developmental quotient $<55$, or major visual or hearing impairment.

Histologically confirmed chorioamnionitis was present in 16 cPVL infants (50\%). A further two infants, for whom placental histology was missing, had $\geq 3$ markers of clinical chorioamnionitis: leukocytes $>20,000 / \mu \mathrm{L}$, $\mathrm{CRP}>40 \mathrm{mg} / \mathrm{dL}$, maternal temperature $>38^{\circ} \mathrm{C}$, maternal tachycardia > $100 \mathrm{bpm}$, and fetal tachycardia > $160 \mathrm{bpm}$. Thus, $18 / 32$ cases (56\%) were classified as having been complicated by chorioamnionitis. Two cases were assigned to the nonchorioamnionitis group despite the absence of placental histology and a number of clinical parameters.

The control group included 134 cases of suspected chorioamnionitis. Review of the placental histology and clinical parameters reduced these to 71 cases of histological chorioamnionitis and two cases of clinical chorioamnionitis in the absence of placental histology. Chorioamnionitis thus complicated 73/6440 (1.1\%) of control deliveries.

Preliminary logistic regression revealed significant associations between CPVL and chorioamnionitis, male sex, and birth weight. Chorioamnionitis had the highest impact on cPVL risk (OR 35.9, 95\% CI 12.6-102.7). However, because a prenatal diagnosis of chorioamnionitis is often not possible, logistic regression was performed, replacing chorioamnionitis by the pPROM-delivery interval. This revealed significant impacts on cPVL by sex $(P=0.008)$, pPROM-delivery interval $>48$ hours $(P<0.001)$, and fetal weight $(P<0.001$; Table 3). 
TABLE 3: Multivariate logistic regression analysis of the influence of fetal sex, preterm premature rupture of the membranes- (pPROM-) delivery interval, and birth weight on cystic periventricular leukomalacia (cPVL) prevalence.

\begin{tabular}{lcc}
\hline Covariate & cPVL & $P$ \\
\hline Sex & & \\
$\quad$ Female & \\
$\quad$ Male & 1 & \\
pPROM-delivery interval & $3.1(1.3-7.1)$ & 0.008 \\
$\quad \leq 48 \mathrm{~h}^{*}$ & & \\
$\quad>48 \mathrm{~h}$ & 1 & \\
Per 100 g higher fetal weight & $0.85(0.81-0.89)$ & $<0.001$ \\
\hline
\end{tabular}

Data are adjusted odds ratios ( $95 \%$ confidence intervals).

* Baseline category.

TABLE 4: Regression analysis of the impact of different birth weights and preterm premature rupture of membranes- (pPROM-) delivery intervals on periventricular leukomalacia risk, assuming fetal weight gains of $0 \mathrm{~g}$ in $24 \mathrm{~h}$ and $48 \mathrm{~h}, 200 \mathrm{~g}$ in 1 week, and $400 \mathrm{~g}$ in 2 weeks [26]. Data are estimated incidence of periventricular leukomalacia.

(a) Male infants

\begin{tabular}{llllll}
\hline & \multicolumn{5}{c}{ Birth weight } \\
& $500 \mathrm{~g}$ & $1000 \mathrm{~g}$ & $1500 \mathrm{~g}$ & $2000 \mathrm{~g}$ & $2500 \mathrm{~g}$ \\
\hline pPROM-delivery interval & & & & & \\
$0 \mathrm{~h}$ & 0.268 & 0.096 & 0.035 & 0.012 & 0.004 \\
$24 \mathrm{~h}$ & 0.279 & 0.100 & 0.036 & 0.013 & 0.005 \\
$48 \mathrm{~h}$ & 0.291 & 0.105 & 0.038 & 0.014 & 0.005 \\
1 week & 0.240 & 0.086 & 0.031 & 0.012 & 0.004 \\
2 weeks & 0.214 & 0.077 & 0.028 & 0.010 & 0.004 \\
\hline
\end{tabular}

(b) Female infants

\begin{tabular}{|c|c|c|c|c|c|}
\hline & \multicolumn{5}{|c|}{ Birth weight } \\
\hline & $500 \mathrm{~g}$ & $1000 \mathrm{~g}$ & $1500 \mathrm{~g}$ & $2000 \mathrm{~g}$ & $2500 \mathrm{~g}$ \\
\hline \multicolumn{6}{|c|}{ pPROM-delivery interval } \\
\hline $0 \mathrm{~h}$ & 0.081 & 0.029 & 0.010 & 0.004 & 0.001 \\
\hline $24 \mathrm{~h}$ & 0.085 & 0.030 & 0.011 & 0.004 & 0.001 \\
\hline $48 \mathrm{~h}$ & 0.088 & 0.032 & 0.011 & 0.004 & 0.001 \\
\hline 1 week & 0.073 & 0.026 & 0.009 & 0.003 & 0.001 \\
\hline 2 weeks & 0.065 & 0.023 & 0.008 & 0.003 & 0.001 \\
\hline
\end{tabular}

Further multiple logistic regression analyses revealed significant associations between chorioamnionitis and PPROMdelivery interval $>24$ hours $(P=0.002)$ and gestational age $(P<0.001)$. No significant influence of ethnicity $(P=0.49)$, fetal weight $(P=0.37)$, or parity $(P=0.79)$ was observed.

According to logistic regression analyses tabulation of estimated CPVL incidence at varying pPROM-delivery intervals and birth weights for boys and girls (Table 4), assuming fetal weight gain of $200 \mathrm{~g} /$ week [26], revealed a slight rise in the first 48 hours, followed by a significantly lower risk after the first and second week of prolongation of pregnancy. Increasing fetal weight during pPROM latency had a far stronger protective effect despite a prolonged pPROMdelivery interval being a risk factor for CPVL.

\section{Discussion}

Zurich University Hospital's obstetrics department is a tertiary referral center. This accounts for the high prevalence of preterm deliveries compared to the national average $(19 \%$ versus 9\%) [27]. The prevalence of cPVL in our study group $(0.99 \%$ ) appears lower than the few reports in the literature. Hamrick et al. reported an incidence of $1.8 \%$ at UC San Francisco in 1992, falling to $0.2 \%$ in 2002 ; the incidence of cPVL in children weighing $<1500 \mathrm{~g}$ decreased from $2.9 \%$ to $0.5 \%$ over the same period [28].

The difference may be partly due to Zurich's conservative management of newborns below $250 / 7$ weeks of gestation (restriction to comfort care in the majority of cases) [29]. We may also have missed some cases of late cPVL diagnosis in children born after 32 0/7 weeks (there were no instances of late diagnosis of brain lesions in preterms included in long-term follow-up). Given our small sample size, we could only extrapolate cPVL incidence for birth weights $<1000 \mathrm{~g}$ (Figure 1). The Vermont Oxford Network reported approximately $3 \% \mathrm{cPVL}$ at birth weights 751-1500 g; risk was highest $(6 \%)$ at birth weights $<751 \mathrm{~g}$ [30]. Our data confirm the reported exponential decrease in cPVL incidence with increasing birth weight $[28,30]$. They also confirm a similar exponential decrease with advancing gestational age independently of birth weight [30]. Again, our data at below $260 / 7$ weeks are not comparable to other centers due to our conservative management of newborns around $250 / 7$ weeks.

Our data support the dependency of cPVL risk on the pPROM-delivery interval $[8,31]$, but not on either low parity or PROM $[8,10,31]$. The finding of a $4: 1$ male/female ratio confirms previous reports $[8,15,31]$ but remains unelucidated.

We also confirmed the several reports of a significant association between CPVL and chorioamnionitis $[8,9,11,14$, 15]. The fact that only one cPVL baby had a positive blood culture within 3 days of birth supports the hypothesis that fetal inflammatory response syndrome is perfectly capable of causing brain damage even in the absence of bacteremia [3234]. Apart from being a risk factor for CPVL, chorioamnionitis is well-recognized as correlating with neonatal morbidity and mortality [32-34].

Unfortunately, these risk factors cannot resolve our strategic dilemma of immediate versus delayed delivery for lowering short-term mortality and long-term sequelae. Chorioamnionitis is a major complication of pPROM but probably even more often the cause of pPROM.

The increase in CPVL risk during the first 48 hours after pPROM and the substantial decrease thereafter at varying birth weights and pPROM-delivery intervals (Table 4) can be interpreted in several ways. For example, the initial increase may relate to the use of antenatal steroids to induce lung maturation. Steroids could facilitate the spread of bacterial infection; they could also modulate the fetal inflammatory cytokines thought to cause brain damage [14]. Corroborative evidence is that the incidence of cPVL in our group decreased from $1.3 \%$ on repeated steroid courses to $0.7 \%$ on singlecourse steroids (although we must admit to having concomitantly switched to erythromycin and introduced nifedipine 


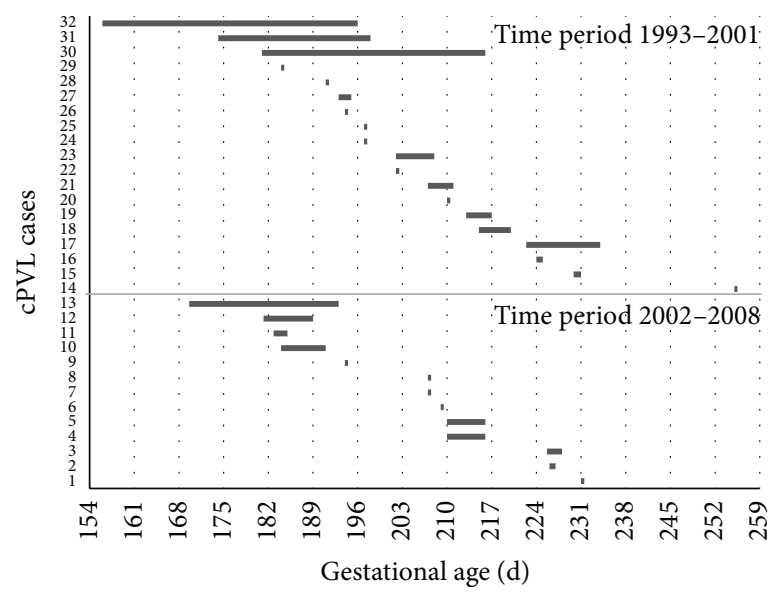

FIGURE 2: Data from 32 cPVL cases in two time periods. Each bar represents a pregnancy with pPROM-delivery interval beginning with gestational age of $\mathrm{pPROM}$.

tocolysis). This study could not add any information about the impact of the different changed interventions to the lower cPVL incidence 2002-2008.

Another explanation is that the inverted U-shaped risk for $\mathrm{cPVL}$ with respect to the pPROM-delivery interval results from superposing two distinct groups of women: one with PPROM due to chorioamnionitis and the other with pPROM from a noninfectious cause. Most chorioamniotic pregnancies will deliver within a few days after PPROM. Thus, women still pregnant after one week of pPROM are more likely to have a noninfectious cause of fluid leakage. As a consequence, delaying delivery in these cases would lower cPVL risk by allowing birth weight to increase. At the same time, by increasing gestational age and birth weight, this strategy would substantially decrease all other complications of prematurity such as cerebral hemorrhage or lung pathology [35]. Conversely, a conservative strategy would increase cPVL risk in the infectious group. An increased risk for neurodevelopmental impairment in the first 48 to 72 hours after pPROM was also observed in a large French cohort with 1884 infants born at 24-32 weeks of gestational age [36].

Given this strong association between CPVL and chorioamnionitis, it is absolutely essential to diagnose intrauterine infection as early as possible. This points once again to the urgent need for a tool that reliably diagnoses chorioamnionitis.

In the absence of such a tool, either strategy carries a considerable risk of Pyrrhic victory. Because cPVL affects only a small proportion of newborns, with other problems of prematurity playing a much larger role, it seems not unreasonable to pursue a conservative strategy until prospective randomized trials provide a definitive answer or, at the least, until we have a reliable test for the early diagnosis of chorioamnionitis. Perinatal morbidity is strongly correlated with prematurity and latency does not appear to worsen outcome in PPROM [37].

The fact that chorioamnionitis and the pPROM-delivery interval seem to have a high impact on the risk of developing
cPVL in our study could have important consequences for the future management of pPROM.

The strengths of this study are that all infants and mothers were monitored in the same department and that all preterms below 32 0/7 weeks received long-term follow-up, where possible (the actual follow-up rate was $89 \%$ ). Only in midstudy were there any relevant changes in the management of pPROM (antibiotics, tocolytic, and steroid courses).

The study's limitations lie in its retrospective design and small cPVL sample size. We had particularly few birth weights below $1000 \mathrm{~g}$, partly because the Swiss Society of Neonatology recommends restricting neonatal management to comfort care at gestational ages below 24 0/7 weeks. Between 24 0/7 and $256 / 7$ weeks of gestation the decision to undertake intensive care is individual and influenced by prenatal factors such as birth weight, gender, antenatal steroid use, intrauterine growth restriction, chorioamnionitis, fetal malformation, multiple gestation, and clinical condition immediately after delivery (asphyxia, heart rate, activity, and response to initial resuscitation). Intervention continues in the neonatal intensive care unit with the primary goal of survival with an acceptable quality of life [29]. Our results are helpful for deliveries between 26 and 34 weeks of gestation only.

Other factors accounting for small sample size in any study of this kind include the fact that the true number of newborns with cPVL can be difficult to evaluate due to early postnatal death, especially when gestational age is very low. cPVL can often only be diagnosed weeks after birth because it takes time for the periventricular cysts to become visible on ultrasound. Failure of underreporting when making a late diagnosis of $\mathrm{CPVL}$ is another source.

Even if a prolonged pPROM-delivery interval may briefly increase the risk of $\mathrm{CPVL}$, we believe that conservative management makes sense in the absence of clinical chorioamnionitis. Higher infant weight at delivery compensates for the impact of pPROM latency on neonatal outcome provided that the pregnancy can be prolonged by more than 48 hours.

\section{Conflict of Interests}

The authors declare that they have no financial or nonfinancial conflict of interests.

\section{Authors' Contribution}

Annick Denzler and Tilo Burkhardt have made substantial contributions to acquisition of obstetrical data and analysis and interpretation of all data. Giancarlo Natalucci has substantial contributions to acquisition of neonatal and outcome data. Roland Zimmermann conceived of the study and participated in coordination and helped to draft the paper. All authors read and approved the final paper. Annick Denzler and Tilo Burkhardt contributed equally to the study and paper.

\section{References}

[1] F. Stanley, E. Blair, and E. Alberman, "Pathways to cerebral palsy involving very preterm birth," in Cerebral Palsies Epidemiology 
\& Causal Pathways, F. Stanley, E. Blair, and E. Alberman, Eds., pp. 60-82, Mac Keith Press, London, UK, 2000.

[2] K. Himmelmann, G. Hagberg, E. Beckung, B. Hagberg, and P. Uvebrant, "The changing panorama of cerebral palsy in Sweden. IX. Prevalence and origin in the birth-year period 1995-1998," Acta Paediatrica, vol. 94, no. 3, pp. 287-294, 2005.

[3] S. Winter, A. Autry, C. Boyle, and M. Yeargin-Allsopp, "Trends in the prevalence of cerebral palsy in a population-based study," Pediatrics, vol. 110, no. 6, pp. 1220-1225, 2002.

[4] A. Leviton and N. Paneth, "White matter damage in preterm newborns: an epidemiologic perspective," Early Human Development, vol. 24, no. 1, pp. 1-22, 1990.

[5] J. J. Volpe and A. Zipurksy, "Neurobiology of periventricular leukomalacia in the premature infant," Pediatric Research, vol. 50, no. 5, pp. 553-562, 2001.

[6] Y. Murata, A. Itakura, K. Matsuzawa, A. Okumura, K. Wakai, and S. Mizutani, "Possible antenatal and perinatal related factors in development of cystic periventricular leukomalacia," Brain \& Development, vol. 27, no. 1, pp. 17-21, 2005.

[7] I. Blumenthal, "Periventricular leucomalacia: a review," European Journal of Pediatrics, vol. 163, no. 8, pp. 435-442, 2004.

[8] M. M. Costantine, H. Y. How, K. Coppage, R. A. Maxwell, and B. M. Sibai, "Does peripartum infection increase the incidence of cerebral palsy in extremely low birthweight infants?" The American Journal of Obstetrics and Gynecology, vol. 196, no. 5, pp. e6-e8, 2007.

[9] R. Romero, J. Espinoza, L. F. Gonçalves, J. P. Kusanovic, L. Friel, and S. Hassan, "The role of inflammation and infection in preterm birth," Seminars in Reproductive Medicine, vol. 25, no. 1, pp. 21-39, 2007.

[10] Y. W. Wu, G. J. Escobar, J. K. Grether, L. A. Croen, J. D. Greene, and T. B. Newman, "Chorioamnionitis and cerebral palsy in term and near-term infants," Journal of the American Medical Association, vol. 290, no. 20, pp. 2677-2684, 2003.

[11] K. Tsukimori, H. Komatsu, T. Yoshimura et al., "Increased inflammatory markers are associated with early periventricular leukomalacia," Developmental Medicine and Child Neurology, vol. 49, no. 8, pp. 587-590, 2007.

[12] E. M. Graham, C. J. Holcroft, K. K. Rai, P. K. Donohue, and M. C. Allen, "Neonatal cerebral white matter injury in preterm infants is associated with culture positive infections and only rarely with metabolic acidosis," The American Journal of Obstetrics and Gynecology, vol. 191, no. 4, pp. 1305-1310, 2004.

[13] A. Bashiri, E. Burstein, and M. Mazor, "Cerebral palsy and fetal inflammatory response syndrome: a review," Journal of Perinatal Medicine, vol. 34, no. 1, pp. 5-12, 2006.

[14] G. Rocha, E. Proença, C. Quintas, T. Rodrigues, and H. Guimaríes, "Chorioamnionitis and brain damage in the preterm newborn," Journal of Maternal-Fetal and Neonatal Medicine, vol. 20, no. 10, pp. 745-749, 2007.

[15] E. Hatzidaki, E. Giahnakis, S. Maraka et al., "Risk factors for periventricular leukomalacia," Acta Obstetricia et Gynecologica Scandinavica, vol. 88, no. 1, pp. 110-115, 2009.

[16] B. M. Mercer, "Preterm premature rupture of the membranes," Obstetrics and Gynecology, vol. 101, no. 1, pp. 178-193, 2003.

[17] RCOG, "Guideline No. 44: Preterm prelabour rupture of membranes," November 2006.

[18] S. Buchanan, C. Crowther, and J. Morris, "Preterm prelabour rupture of the membranes: a survey of current practice," The Australian and New Zealand Journal of Obstetrics and Gynaecology, vol. 44, no. 5, pp. 400-403, 2004.
[19] L. Hartling, R. Chari, C. Friesen, B. Vandermeer, and T. LacazeMasmonteil, "A systematic review of intentional delivery in women with preterm prelabor rupture of membranes," Journal of Maternal-Fetal and Neonatal Medicine, vol. 19, no. 3, pp. 177187, 2006.

[20] J. C. Pasquier, E. Bujold, M. Rabilloud et al., "Effect of latency period after premature rupture of membranes on 2 years infant mortality (DOMINOS study)," European Journal of Obstetrics Gynecology and Reproductive Biology, vol. 135, no. 1, pp. 21-27, 2007.

[21] S. L. Buchanan, C. A. Crowther, K. M. Levett, P. Middleton, and J. Morris, "Planned early birth versus expectant management for women with preterm prelabour rupture of membranes prior to 37 weeks' gestation for improving pregnancy outcome," The Cochrane Database of Systematic Reviews, no. 3, Article ID CD004735, 2010.

[22] AMWF-Leitlinien-Register: Empfehlungen zum Vorgehen bei vorzeitigem Blasensprung, http://www.awmf.org/.

[23] L. S. de Vries, P. Eken, and L. M. S. Dubowitz, "The spectrum of leukomalacia using cranial ultrasound," Behavioural Brain Research, vol. 49, no. 1, pp. 1-6, 1992.

[24] S. L. Kenyon, D. J. Taylor, and W. Tarnow-Mordi, "Broadspectrum antibiotics for preterm, prelabour rupture of fetal membranes: the ORACLE I randomised trial," The Lancet, vol. 357, no. 9261, pp. 979-988, 2001.

[25] R. Palisano, P. Rosenbaum, S. Walter, D. Russell, E. Wood, and B. Galuppi, "Development and reliability of a system to classify gross motor function in children with cerebral palsy," Developmental Medicine \& Child Neurology, vol. 39, no. 4, pp. 214-223, 1997.

[26] G. M. Beutler, J. Kurmanaviclus, M. Hoffmann, E. Welzl, R. Huch, and M. Bajka, "New nomogram for foetal weight estimation based on Hadlock's two-parameter formula," Ultraschall in der Medizin, vol. 25, no. 1, pp. 58-64, 2004.

[27] Bundesamt für Statistik (BFS), Neugeborene in Schweizer Spitälern 2004, Stat Santé, 2004.

[28] S. E. G. Hamrick, S. P. Miller, C. Leonard et al., "Trends in severe brain injury and neurodevelopmental outcome in premature newborn infants: the role of cystic periventricular leukomalacia," Journal of Pediatrics, vol. 145, no. 5, pp. 593-599, 2004.

[29] T. Berger, V. Büttiker, J. Fauchère et al., "ecommendations for the care of infants born at the limit of viability (gestational age 22-26 weeks)," in Swiss Society of Neonatology, 2002.

[30] Vermont Oxford Network, Vermont-Oxford Network Expanded Database Summary, Burlington, Burlington, Vt, USA, 2006.

[31] M. Bauer, C. Fast, J. Haas, B. Resch, U. Lang, and B. Pertl, “Cystic periventricular leukomalacia in preterm infants: an analysis of obstetric risk factors,' Early Human Development, vol. 85, no. 3, pp. 163-169, 2009.

[32] J. M. Zhang, F. T. Kraus, and T. I. Aquino, "Chorioamnionitis: a comparative histologic, bacteriologic, and clinical study," International Journal of Gynecological Pathology, vol. 4, no. 1, pp. $1-10,1985$.

[33] J. V. Been, I. G. I. J. G. Rours, R. F. Kornelisse et al., "Histologic chorioamnionitis, fetal involvement, and antenatal steroids: effects on neonatal outcome in preterm infants," The American Journal of Obstetrics and Gynecology, vol. 201, no. 6, pp. 587.e1587.e8, 2009.

[34] A. Wolfensberger, R. Zimmermann, and U. Von Mandach, "Neonatal mortality and morbidity after aggressive long-term 
tocolysis for preterm premature rupture of the membranes," Fetal Diagnosis and Therapy, vol. 21, no. 4, pp. 366-373, 2006.

[35] T. P. Waters and B. M. Mercer, "The management of preterm premature rupture of the membranes near the limit of fetal viability," The American Journal of Obstetrics and Gynecology, vol. 201, no. 3, pp. 230-240, 2009.

[36] T. Mura, J.-C. Picaud, B. Larroque et al., "Cognitive impairment at age 5 years in very preterm infants born following premature rupture of membranes," The Journal of Pediatrics, vol. 163, no. 2, pp. 435.e2-440.e2, 2013.

[37] T. A. Manuck, C. C. Maclean, R. M. Silver, and M. W. Varner, "Preterm premature rupture of membranes: does the duration of latency influence perinatal outcomes?" American Journal of Obstetrics \& Gynecology, vol. 201, no. 4, pp. 414.el-414.e6, 2009. 


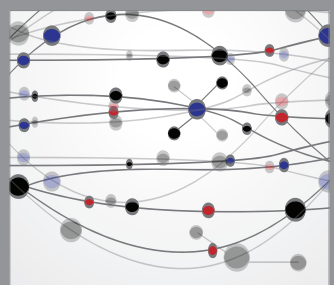

The Scientific World Journal
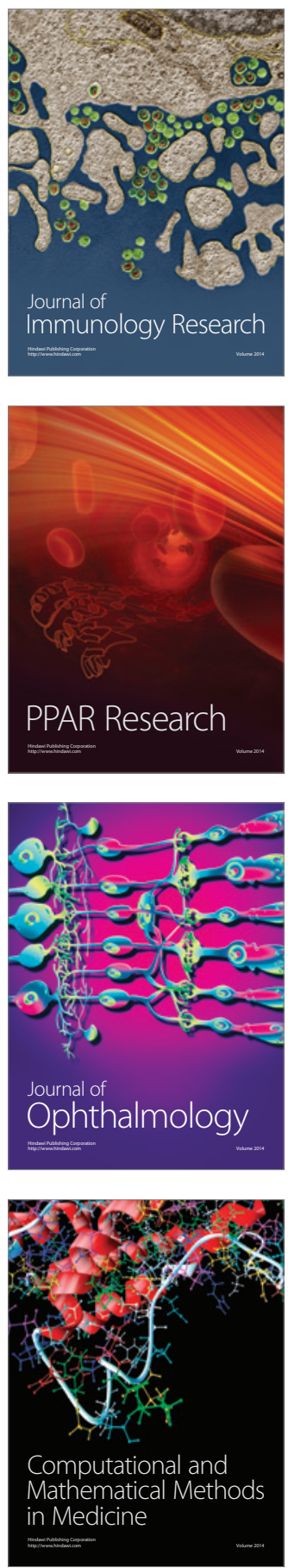

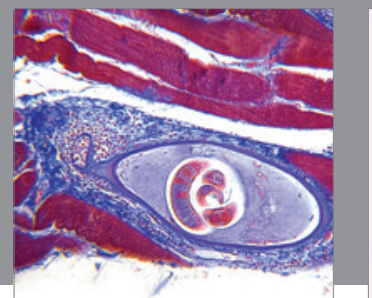

Gastroenterology

Research and Practice
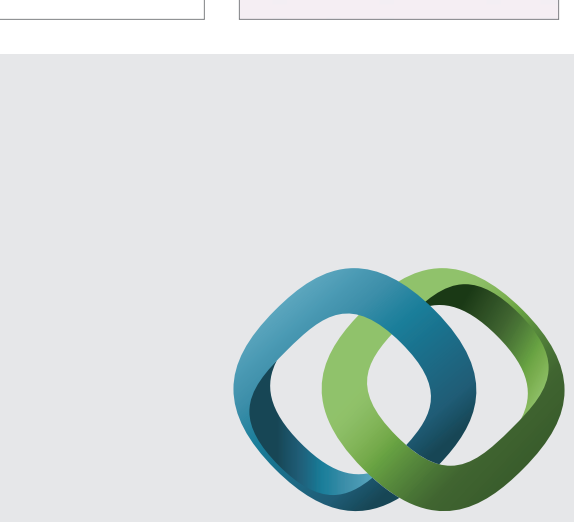

\section{Hindawi}

Submit your manuscripts at

http://www.hindawi.com



Disease Markers
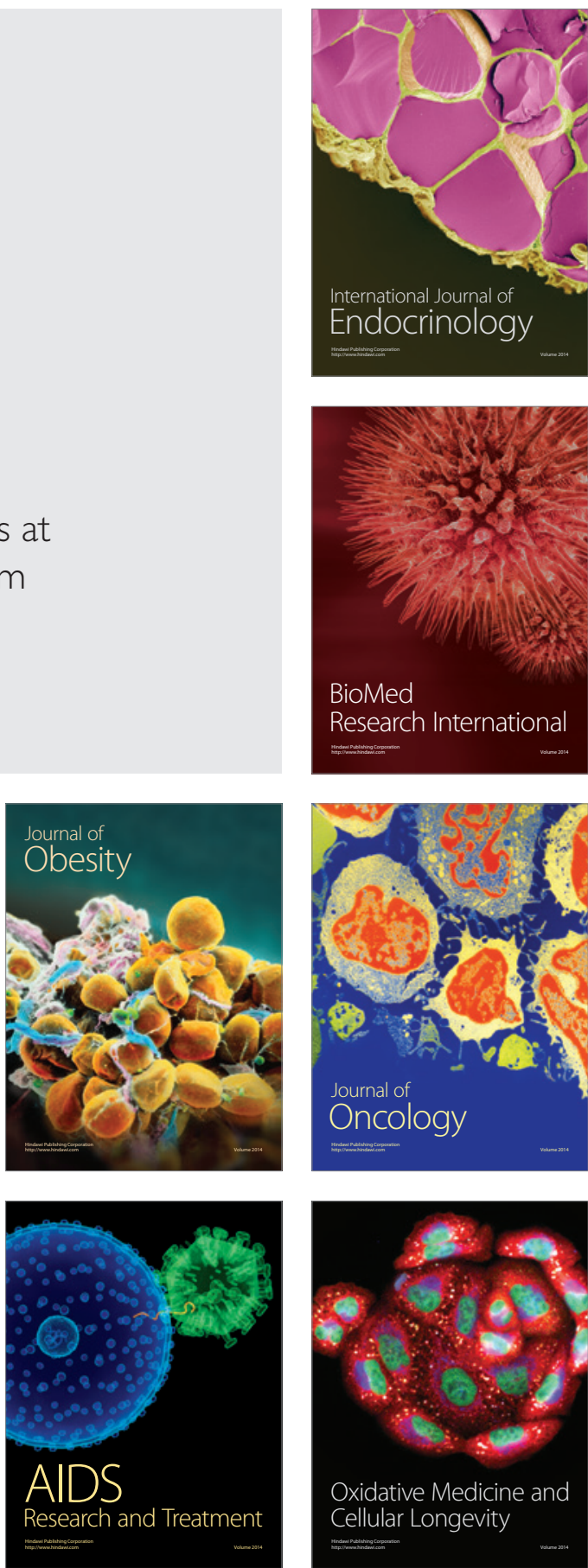